\title{
Ultrasonography-guided modified thoracolumbar interfascial plane block: a new approach
}

\author{
Ali Ahiskalioglu, MD $($ Haci Ahmet Alici, MD • Kubra Selvitopi, MD • \\ Ahmet Murat Yayik, MD
}

Received: 4 January 2017/Revised: 11 February 2017/Accepted: 17 February 2017/Published online: 27 February 2017

(c) Canadian Anesthesiologists' Society 2017

\section{To the Editor,}

A recent study published by Hand et al. described an ultrasonography-guided thoracolumbar interfascial plane (TLIP) block. ${ }^{1}$ This block involves injecting local anesthetics between the multifidus and longissimus muscles at the third lumbar vertebral level to block the dorsal rami of thoracolumbar nerves. ${ }^{2}$ Hand et al. made the injection between the multifidus and longissimus muscles by positioning the block needle at a $30^{\circ}$ angle from the skin and advanced it from a lateral to medial direction (Figure).

Herein, we describe our TLIP modification where we inject the anesthetics between the longissimus and iliocostalis muscles after having advanced the needles at a $15^{\circ}$ angle in a medial to lateral direction. Our modified method has several advantages.

1. Advancing the needle from a medial to lateral direction eliminates the risk of possible inadvertent neuraxial injection.
A. Ahiskalioglu, MD $(\bowtie) \cdot$ K. Selvitopi, MD .

A. M. Yayik, MD

Department of Anesthesiology and Reanimation, Ataturk

University School of Medicine, Erzurum, Turkey

e-mail: aliahiskalioglu@ hotmail.com

H. A. Alici, MD

Department of Pain Clinic, Ataturk University School of Medicine, Erzurum, Turkey
2. Injecting between the iliocostalis and longissimus muscles results in a dermatomal area of analgesia similar to that obtained with an injection made between the multifidus and longissimus muscles. We have demonstrated this area of analgesia using radiopaque dye injections (with specific patient consent) where the distribution of the local anesthetics spread two levels caudal and cranial to the injection site. Twenty minutes after injection, we could show a corresponding dermatomal area (using a pinprick test) of reduced sensation.

3. Sonographic imaging more easily discerns the distinction between the longissimus and iliocostalis muscles than between the multifidus and longissimus muscles, thereby potentially increasing the success rate of the block.

These modifications to the TLIP block will require further study to investigate their potential advantages more fully. Such investigations, including magnetic resonance imaging and cadaveric studies combined with ultrasonography, should be beneficial for evaluating the block's potential anatomic spread. When compared with some other nerve blocks, interfascial plane blocks are highly dependent on sufficient volumes of local anesthetics to spread between the muscle layers and fascial planes. The TLIP block might be useful for 2 and 3 vertebral level spinal surgical procedures as well as minimally invasive spinal surgery. 


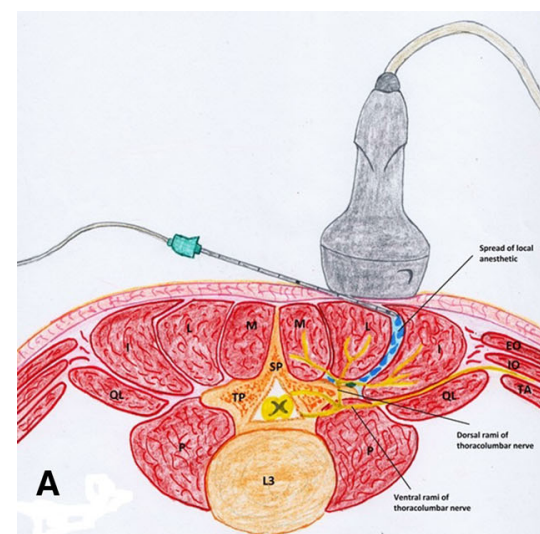

Figure The modified thoracolumbar interfascial plane block is accomplished by advancing the block needle at a $15^{\circ}$ angle (from the skin) in a medial to lateral direction (image A) and injecting the anesthetic locally between the longissimus and intercostalis muscles, which are easy to define using ultrasonography (image B). Injection

Conflicts of interest The authors declare that there were A) no funding sources (departmental, hospital, institutional, commercial) to support the submitted work; B) no commercial or non-commercial affiliations that are, or could be perceived as, a conflict of interest for the work of each author; and C) no other financial associations, such as consultancies.

Editorial responsibility This submission was handled by Dr. Hilary P. Grocott, Editor-in-Chief, Canadian Journal of Anesthesia. of radiographic dye shows multilevel spread of the injected local anesthetic (image C). EO = external oblique; $\mathrm{I}=$ iliocostalis; $\mathrm{IO}=$ internal oblique, $\mathrm{L}=$ longissimus; $\mathrm{L} 3=$ lumbar third vertebra; $\mathrm{M}=$ multifidus; $\mathrm{P}=$ psoas, $\mathrm{QL}=$ quadratus lumborum; $\mathrm{SP}=$ spinous process; $\mathrm{TA}=$ transversus abdominis; $\mathrm{TP}=$ transverse process

\section{References}

1. Hand WR, Taylor JM, Harvey NR, et al. Thoracolumbar interfascial plane (TLIP) block: a pilot study in volunteers. Can J Anesth 2015; 62: 1196-200.

2. Ueshima $H$, Oku K, Otake H. Ultrasound-guided thoracolumbar interfascial plane block: a cadaveric study of the spread of injectate. J Clin Anesth 2016; 34: 259-60. 